\title{
Theoretical models of neutrino-nucleus cross sections
}

\author{
Marco Martini*i† \\ Department of Physics and Astronomy, Ghent University, Proeftuinstraat 86, B-9000 Gent, \\ Belgium \\ E-mail: martini.marco@gmail.com
}

In the present and future accelerator-based neutrino oscillation experiments nuclear targets (such as $\mathrm{C}, \mathrm{O}, \mathrm{Ar}$ and $\mathrm{Fe}$ ) are involved. Hence the knowledge of neutrino-nucleus scattering is very important. In particular it is crucial for the determination of the neutrino energy which enters the expression of the oscillation probability, since this energy is reconstructed from the final states of the neutrino-nucleus reaction. The status of the different theoretical approaches treating the open channels in the few-GeV region, i.e. the quasielastic, the pion production and the multinucleon emission, is reviewed. Special emphasis is devoted to the multinucleon emission channel, which turned to be crucial to explain the unexpected behavior of the charged current quasielastic measurement performed by MiniBooNE. Up to last year, this channel was not included in the generators used for the analyses of the neutrino cross sections and oscillations experiments. The theoretical and experimental interest towards these multinucleon excitations continues to increase.

The European Physical Society Conference on High Energy Physics

22-29 July 2015

Vienna, Austria

\footnotetext{
* Speaker.

$\dagger$ I thank the conveners of the Neutrino Physics parallel session for the opportunity to present this review and Magda Ericson for a continuous collaboration and for numerous discussions. The financial support of the Interuniversity Attraction Poles Programme initiated by the Belgian Science Policy Office (BriX network P7/12) is acknowledged.
} 


\section{Introduction}

Neutrino physics has undergone a spectacular development in the last decade, following the discovery of neutrino oscillations. In the present and future accelerator-based neutrino oscillation experiments nuclear targets, such as ${ }^{12} \mathrm{C},{ }^{16} \mathrm{O}{ }^{40} \mathrm{Ar}$ or ${ }^{56} \mathrm{Fe}$, are involved, hence the knowledge of neutrino-nucleus scattering is very important. In particular it is crucial for the determination of the neutrino energy which enters the expression of the oscillation probability. In accelerator-based experiments the neutrino beams (at difference with respect to electron beams, for example) are not monochromatic but they span a wide range of energies, hence the incoming neutrino energy is reconstructed from the final states of the reaction. This determination is typically done through the charged current quasielastic (CCQE) events, commonly defined as those in which the emission product only includes one charged lepton. The reconstructed energy hypothesis used to obtain the neutrino energy from the measured charged lepton variables (energy and scattering angle) via a two-body formula is that the neutrino interaction in the nuclear target takes place on a nucleon at rest. The identification of the reconstructed neutrino energy with the real one is too crude. Several nuclear effects such as Pauli blocking, Fermi motion, collective aspects of the nuclear response and, very important, multinucleon emission, need to be taken into account. Furthermore, the fact that in the accelerator-based experiments the incoming neutrino beam exhibits a wide spectrum of energies implies that not only the quasielastic but also other reaction mechanisms, such as for example the pion production, contribute to the neutrino nucleus cross section. The status of the different theoretical approaches treating the open channels in the few-GeV region, i.e. the quasielastic, the pion production and the multinucleon emission, is here reviewed, devoting special emphasis to the multinucleon emission channel.

\section{Quasielastic and multinucleon emission}

The multinucleon emission channel in connection with the quasielastic has attracted a lot of attention in these last years. In fact, as illustrated in Fig. 1, the inclusion of this channel in the quasielastic cross section was suggested $[1,2]$ to be the possible explanation of the MiniBooNE CCQE total cross section on carbon [3], apparently too large with respect to many theoretical predictions employing the standard value of the axial mass. Since the MiniBooNE experiment, as well as other experiments involving Cherenkov detectors, defines a charged current "quasielastic" event as the one in which only a final charged lepton is detected, the ejection of a single nucleon (a genuine quasielastic event) is only one possibility, and one must in addition consider events involving a correlated nucleon pair from which the partner nucleon is also ejected, as discussed first by Marteau et al. in Ref. [4]. This leads to the excitation of 2 particle-2 hole (2p-2h) states; $3 \mathrm{p}-3 \mathrm{~h}$ excitations are also possible. The inclusion in the quasielastic cross section of events in which several nucleons are ejected (np-nh excitations), leads to an excess over the genuine quasielastic value. Martini et al. [1,2] have argued that this is the likely explanation of the MiniBooNE anomaly showing that their evaluation can account for the excess in the cross section without any modification of the axial mass. After this suggestion the interest of the neutrino scattering and oscillation communities on the multinucleon emission channel rapidly increased. Indeed this channel was not included in the generators used for the analyses of the neutrino cross sections and 
oscillations experiments. Today there is an effort to include this np-nh channel in several Monte Carlo. Concerning the theoretical situation, nowadays several calculations agree on the crucial role of the multinucleon emission in order to explain the MiniBooNE neutrino [3] and antineutrino [5] data as well as the SciBooNE [6] and T2K [7] inclusive cross sections. Nevertheless there are some differences on the results obtained for this np-nh channel by the different theoretical approaches. The aim of this section is to review the current theoretical status on this subject.

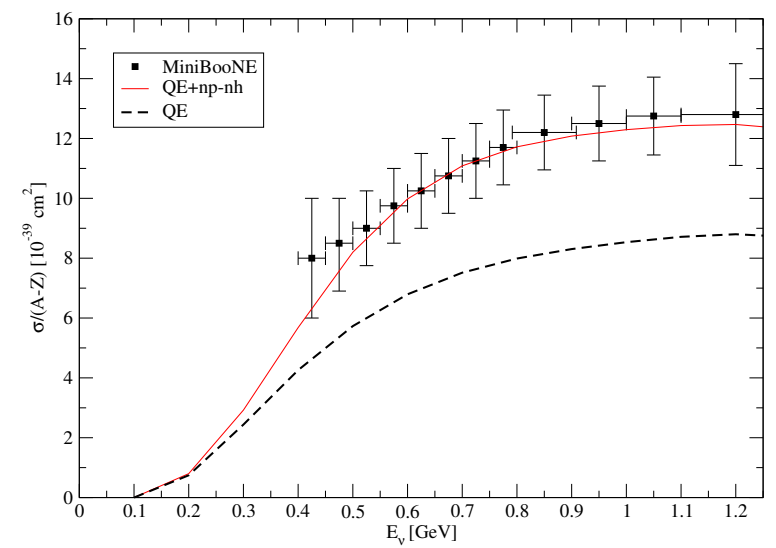

Figure 1: Charged current "Quasielastic" cross section on carbon measured by MiniBooNE [3] compared to Martini et al. calculations. The figure is taken from Ref. [1].

The theoretical calculations of np-nh excitations contributions to neutrino-nucleus cross sections are actually performed essentially by three groups. There are the works of Martini et al. $[1,2,8,9,10,11,12,13]$, the ones of Nieves et al. $[14,15,16,17,18]$ and the ones of Amaro et al. [19, 20, 21, 22, 23, 24]. In the 2p-2h sector, these three approaches are based on the Fermi gas. We remind that there exist several two-body current contributions. We have first of all the Meson Exchange Currents (MEC), given by the pion-in-flight term, the contact term and the $\Delta$ intermediate state or $\Delta$-MEC term. Furthermore in the independent particle models, such as the Fermi gas, the nucleon-nucleon (NN) correlation must be taken into account. It is possible by including an additional two-body current, the correlation current. Even in the simple Fermi gas model, an exact relativistic calculation of all the two-body current contributions is difficult for several reasons. First of all it involves the computation of 7-dimensional integrals for a huge number of $2 \mathrm{p}-2 \mathrm{~h}$ response Feynman diagrams. Second divergences in the NN correlations sector and in the angular distribution of the ejected nucleons $[22,23]$ may appear and need to be regularized. Furthermore the calculations should be performed for all the kinematics compatible with the experimental neutrino flux. For these reasons an exact relativistic calculation is computationally very demanding and as a consequence different approximations are employed by the different groups in order to reduce the dimension of the integrals and to regularize the divergences. The choice of subset of diagrams and terms to be calculated presents also important differences. In this connection Amaro et al. explicitely add to the genuine quasielastic only the MEC contributions and not the NN correlations-MEC interference terms. MEC contributions, NN correlations and NN correlationsMEC interference are present both in Martini et al. and Nieves et al. even if Martini et al. consider only the $\Delta$-MEC. On the other hand the treatment of Amaro et al. is fully relativistic as well as 
the one of Nieves et al. while the results of Martini et al. are related to a non-relativistic reduction of the two-body currents. The interference between direct and exchange diagrams is neglected by Martini et al. and Nieves et al. Another important difference is that Amaro et al. consider the $2 \mathrm{p}-2 \mathrm{~h}$ contribution only in the vector sector while Martini et al. and Nieves et al. also in the axial one. Fully relativistic calculations of Amaro et al. for the axial sector are in progress. Taking into account the existence of all these differences, is not surprising that these models produce different final results. This point is illustrated in Fig. 2 where the MiniBooNE neutrino and antineutrino flux folded double differential CCQE-like cross sections calculated in the different approaches are displayed. For sake of illustartion the results are given for $0.8<\cos \theta<0.9$ as function of the muon kinetic energy. The complete theoretical results in the different bins for neutrinos and antineutrinos are given in Refs. [8, 11] for Martini et al., in Refs. [15, 17] for Nieves et al. and in Refs. [19, 21] for Amaro et al. An updated version of these last results is given by Megias et al. in Ref. [24] from which we take the results reported in the last two panels of Fig. 2. As one can observe from Fig. 2 the results of Martini et al. are in good agreement with the experimental data. In the case of Nieves et al. and Amaro et al. there is a tendency to underestimate the MiniBooNE data. Nevertheless also these results are compatible with MiniBooNE since an additional normalization error of $10 \%$ for neutrino and $17 \%$ for antineutrino is not shown in the error bars. An important point is that the relative role of the multinucleon contribution is different for neutrino and antineutrino in the different approaches. The nuclear cross-section difference for neutrinos and antineutrinos stands as a potential obstacle in the interpretation of experiments aimed at the measurement of the $\mathrm{CP}$ violation angle, hence has to be fully mastered. As discussed in Refs. [2, 21, 13] the difference between the neutrino and antineutrino results is due to the presence in the neutrino-nucleus cross section expression of the vector-axial interference term, which changes sign between neutrino and antineutrino, the basic asymmetry which follows from the weak interaction theory. Due to this vector-axial interference term, the relative weight of the different nuclear responses is different for neutrino and antineutrino. As a consequence also the relative weight of the np-nh contributions is different for neutrino and antineutrino. For example the fact that np-nh contributions are larger for antineutrinos with respect to neutrinos in the case of Amaro et al. is due to the fact that Amaro et al. consider the np-nh contributions only in the vector sector, hence not in the vector-axial interference term. In order to investigate the multinucleon content of the vector-axial interference term, Ericson and Martini have recently considered [13] the difference between the neutrino and antineutrino MiniBooNE quasielastic-like double-differential cross sections. They have shown that the model of Martini et al., which includes the np-nh excitations in the vector-axial interference term, gives a good fit for the difference of the MiniBooNE cross sections reproducing well the data in the full range of muon energy and emission angle. This result represents an important test for the presence of the multinucleon component in the vector-axial interference term. A similar conclusion on a relevant two-body current contribution in the vector-axial interference term has been recently obtained by Lovato et al. [25, 26] who calculated the neutral weak current two-body contributions to sum rules and Euclidean responses in ${ }^{12} \mathrm{C}$.

Up to now we have discussed the theoretical models in connection with the MiniBooNE cross sections. For the moment the theoretical calculations for the np-nh excitations are restricted to the relatively small energy and momentum transfer, prevalent in the MiniBooNE and T2K experiments. As already mentioned fully relativistic calculations of Amaro et al. for the axial two-body current 

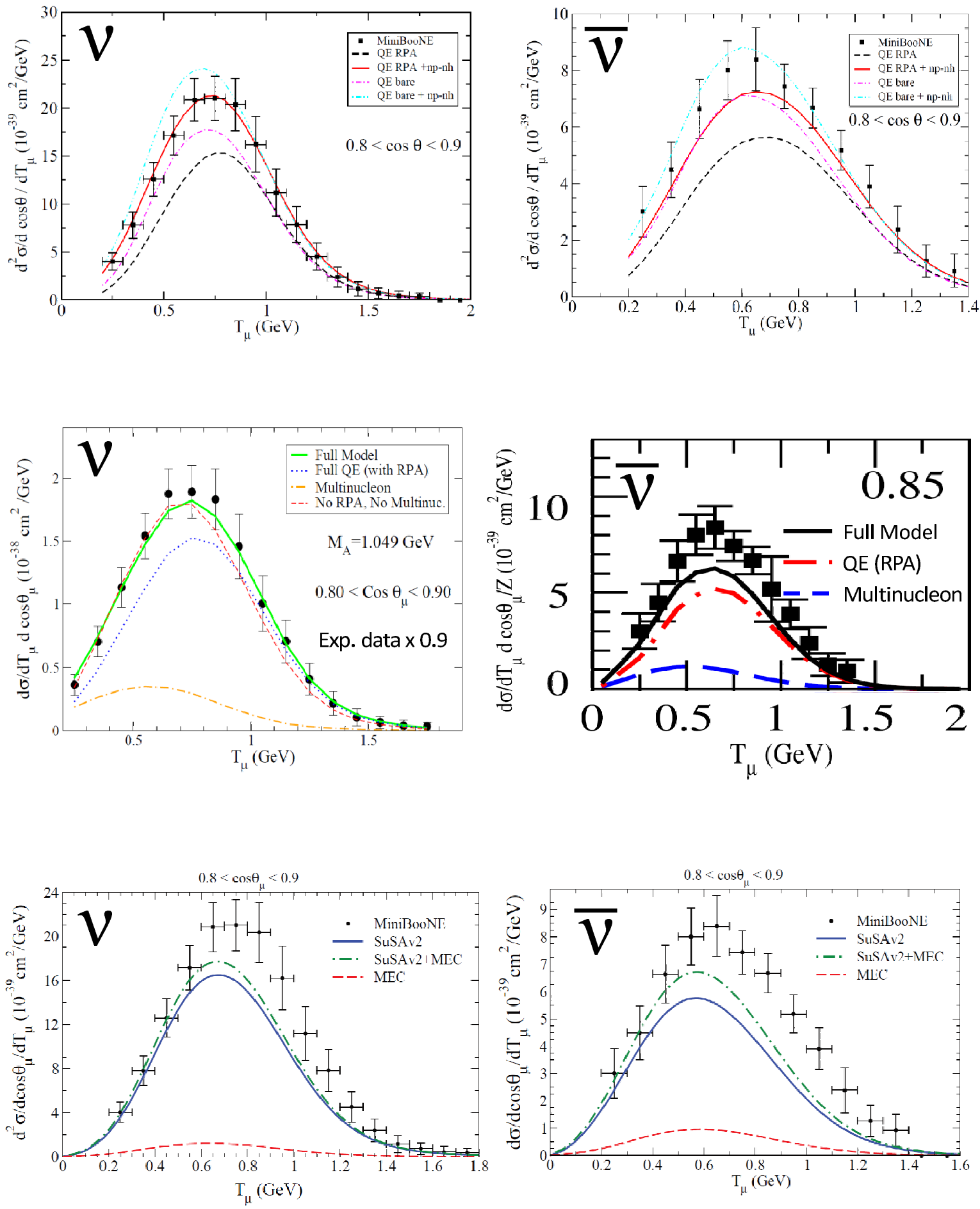

Figure 2: MiniBooNE flux integrated neutrino (left panels) and antineutrino (right panels) CCQE-like double differential cross section on carbon per active nucleon for $0.8<\cos \theta<0.9$ as a function of the muon kinetic energy. Top panels: Martini et al. [8, 11] results. Middle panels: Nieves et al. [15, 17] results. Bottom panels: Megias et al. [24] results representing an update of the Amaro et al. [19, 21] results. 
contributions are still in progress. How the np-nh processes behave at large energy and momentum transfer is still an open question. Nevertheless Megias et al. [24] applied the model of Amaro et $a l$. to neutrino energies of up to $100 \mathrm{GeV}$ and compared their predictions also with MINERvA neutrino [27] and antineutrino [28] CCQE data. Gran et al. [18] applied the model of Nieves et al. to neutrino energies of up to $10 \mathrm{GeV}$. However they placed a cut on the three- momentum transfer of $1.2 \mathrm{GeV}$. They compared their results with the MINER $v$ A neutrino and antineutrino CCQE $Q^{2}$ distribution. A similar comparison has been performed also by Mosel et al. [29] using GiBUU. As a general remark, by comparing the results of Refs. [18, 24, 29] with MINERvA data, one can observe that the MINER $v$ A $Q^{2}$ distributions can be reproduced also without the inclusion of np-nh excitations. This is not the case of the MiniBooNE $Q^{2}$ distributions $[8,11,24]$. As stressed by Mosel et al., in the case of MINERvA the sensitivity to details of the treatment of np-nh contributions is smaller than the uncertainties introduced by the $Q^{2}$ reconstruction and our insufficient knowledge of pion production. The MINER $v$ A experiment being at higher energies with respect to the MiniBooNE one, the pion production channel becomes in this case more important hence the background subtraction to isolate genuine $\mathrm{CCQE}$ and $2 \mathrm{p}-2 \mathrm{~h}$ events is delicate.

\section{Pion production and inclusive cross sections}

Turning to the other channels, in this section we rapidly discuss the present situation for the pion production and inclusive cross sections. In the one pion production channel some questions are still open. For instance there is a general tendency of theoretical calculations and Monte Carlo results to underestimate the MiniBooNE data [30, 31] and to overestimate the MINERvA ones [32]. Further investigations are needed. We remind the different energies of MiniBooNE and MINERvA neutrino beams.

The inclusive $v_{\mu}$ CC double differential cross section on carbon has been published by $\mathrm{T} 2 \mathrm{~K}$ in Ref. [7]. The inclusive measurements are important because they are less affected by background subtraction with respect to exclusive channels measurements. Martini and Ericson have compared their predictions with the T2K experimental results in Ref. [12]. In this paper they have shown that in order to obtain an agreement with the $\mathrm{T} 2 \mathrm{~K}$ inclusive data one needs to consider not only the genuine quasielastic and the one pion production channels but also the multinucleon excitations. These results represent the first successful test of the necessity of the multinucleon emission channel in an experiment with another neutrino flux with respect to the one of MiniBooNE.

\section{Neutrino energy reconstruction problems and neutrino oscillations}

The neutrino energy reconstruction problem has been already mentioned in the introduction. The determination of the initial neutrino energy is commonly done through the charged current neutrino-nucleus quasielastic-like events. For these events where only the charged lepton is observed, the only measurable quantities are then its direction, i.e., its emission angle $\theta$ with respect to the neutrino beam direction and its energy $E_{l}$ (or kinetic energy $T_{l}$ and momentum $P_{l}$ ). The neutrino energy $E_{v}$ is unknown. The usual reconstruction procedure assumes that we are dealing with a genuine quasielastic event on a nucleon at rest. The quasielastic condition then gives the value $\overline{E_{v}}$ of the reconstructed energy: $\overline{E_{v}}=\frac{E_{l}-m_{l}^{2} /(2 M)}{1-\left(E_{l}-P_{l} \cos \theta\right) / M}$. Several nuclear effects can influence this 


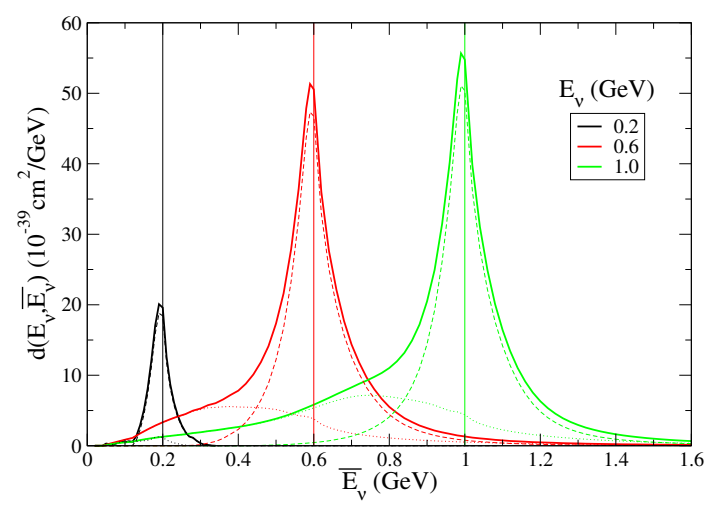

Figure 3: The spreading function per neutron of ${ }^{12} \mathrm{C}$ evaluated for three $E_{v}$ values. The genuine quasielastic (dashed lines) and the multinucleon (dotted lines) contributions are also shown separately. The figure is taken from Ref. [10].

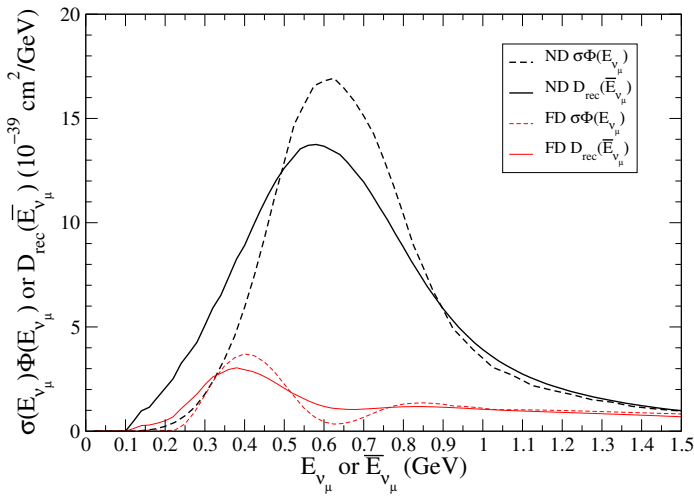

Figure 4: $\quad \mathrm{T} 2 \mathrm{~K}$ distributions per neutrons of muon events before (dashed lines) and after (continuous lines) the energy reconstruction correction in the near and far detector. The figure is taken from Ref. [10].

expression. The most important are the np-nh events which have no reason to fulfill the quasielastic relation. This means that for a given set of lepton variables, $E_{l}$ and $\theta$, an infinity of neutrino energy values, instead of the unique quasielastic value implemented in the neutrino energy reconstruction formula, is possible. Data on neutrino oscillation often involve reconstructed neutrino energies while the analysis implies the real neutrino energy. The corrections corresponding to the transformation from real to reconstructed energy and viceversa are discussed in details in Refs. $[9,10,16,33]$ to which we refer the reader. Here we just summarize some of the main results following the approach of Refs. $[9,10]$. Starting from a theoretical distribution expressed with real energies, i.e. the product of the neutrino cross section $\sigma\left(E_{v}\right)$ by the neutrino energy distribution of the beam $\Phi\left(E_{v}\right)$, a smearing procedure to deduce the corresponding distribution of the events, $D_{\text {rec }}\left(\overline{E_{v}}\right)$, in terms of the reconstructed energy can be performed. This distribution can be expressed in terms of the double differential neutrino-nucleus cross section, according to

$$
D_{\text {rec }}\left(\overline{E_{v}}\right)=\int d E_{v} \Phi\left(E_{v}\right) \int_{E_{l}^{\min }}^{E_{l}^{\max }} d E_{l} \frac{M E_{l}-m_{l}^{2} / 2}{\bar{E}_{v}^{2} P_{l}}\left[\frac{d^{2} \sigma}{d \omega d \cos \theta}\right]_{\omega=E_{v}-E_{l}, \cos \theta=\cos \theta\left(E_{l}, \overline{E_{v}}\right)} .
$$

The second integral on the r.h.s. of Eq. (4.1) is denoted as $d\left(E_{V}, \overline{E_{V}}\right)$. It represents the spreading function and depends on $E_{v}$ and $\overline{E_{v}}$. Some examples of its $\overline{E_{v}}$ dependence for several $E_{v}$ values are given in Fig. 3. As one can observe this spreading function is not symmetrical around $E_{v}$. The multinucleon excitations play a crucial role since they create a low energy tail. Similar results have been obtained in Refs. [16, 33]. In Fig. 4 is shown the application of the smearing procedure of Ref. [10] to the two distributions measured in T2K: the muonic distributions in the near detector (ND) and far detector (FD), hence related to the $v_{\mu}$ disappearance studies. The influence of the reconstructed energy corrections is such that the events tend to escape from the region of high fluxes with a tendency to concentrate at lower energies. Furthermore the middle hole of the far detector results is largely filled. These effects are due to the multinucleon component of the quasielastic-like cross section. Similar results have been obtained in Ref. [33]. The effects are such that an analysis 
which takes into account the smearing effect is likely to lead to some increase of the oscillation mass value.

\section{References}

[1] M. Martini, M. Ericson, G. Chanfray and J. Marteau, Phys. Rev. C 80, 065501 (2009).

[2] M. Martini, M. Ericson, G. Chanfray and J. Marteau, Phys. Rev. C 81, 045502 (2010).

[3] A. A. Aguilar-Arevalo et al. [MiniBooNE Collaboration], Phys. Rev. D 81, 092005 (2010).

[4] J. Marteau, J. Delorme and M. Ericson, Nucl. Instrum. Meth. A 451, 76 (2000).

[5] A. A. Aguilar-Arevalo et al. [MiniBooNE Collaboration], Phys. Rev. D 88, no. 3, 032001 (2013).

[6] Y. Nakajima et al. [SciBooNE Collaboration], Phys. Rev. D 83, 012005 (2011).

[7] K. Abe et al. [T2K Collaboration], Phys. Rev. D 87, 092003 (2013).

[8] M. Martini, M. Ericson and G. Chanfray, Phys. Rev. C 84, 055502 (2011).

[9] M. Martini, M. Ericson and G. Chanfray, Phys. Rev. D 85, 093012 (2012).

[10] M. Martini, M. Ericson and G. Chanfray, Phys. Rev. D 87, 013009 (2013).

[11] M. Martini and M. Ericson, Phys. Rev. C 87, 065501 (2013).

[12] M. Martini and M. Ericson, Phys. Rev. C 90, 025501 (2014).

[13] M. Ericson and M. Martini, Phys. Rev. C 91, no. 3, 035501 (2015).

[14] J. Nieves, I. Ruiz Simo, M. J. Vicente Vacas, Phys. Rev. C83, 045501 (2011).

[15] J. Nieves, I. Ruiz Simo and M. J. Vicente Vacas, Phys. Lett. B 707, 72 (2012).

[16] J. Nieves, F. Sanchez, I. Ruiz Simo and M. J. Vicente Vacas, Phys. Rev. D 85, 113008 (2012).

[17] J. Nieves, I. Ruiz Simo and M. J. Vicente Vacas, Phys. Lett. B 721, 90 (2013).

[18] R. Gran, J. Nieves, F. Sanchez and M. J. Vicente Vacas, Phys. Rev. D 88, 113007 (2013).

[19] J. E. Amaro, M. B. Barbaro, J. A. Caballero, T. W. Donnelly et al. Phys. Lett. B696, 151-155 (2011).

[20] J. E. Amaro, M. B. Barbaro, J. A. Caballero, T. W. Donnelly et al. Phys. Rev. D 84, 033004 (2011).

[21] J. E. Amaro, M. B. Barbaro, J. A. Caballero and T. W. Donnelly, Phys. Rev. Lett. 108, 152501 (2012).

[22] I. Ruiz Simo, C. Albertus, J. E. Amaro, M. B. Barbaro et al. Phys. Rev. D 90, no. 3, 033012 (2014).

[23] I. R. Simo, C. Albertus, J. E. Amaro, M. B. Barbaro et al. Phys. Rev. D 90, no. 5, 053010 (2014).

[24] G. D. Megias, T. W. Donnelly, O. Moreno et al. Phys. Rev. D 91, no. 7, 073004 (2015).

[25] A. Lovato, S. Gandolfi, J. Carlson, S. C. Pieper, R. Schiavilla, Phys. Rev. Lett. 112, 182502 (2014).

[26] A. Lovato, S. Gandolfi, J. Carlson, S. C. Pieper and R. Schiavilla, Phys. Rev. C 91, 062501 (2015).

[27] G. A. Fiorentini et al. [MINERvA Collaboration], Phys. Rev. Lett. 111, 022502 (2013).

[28] L. Fields et al. [MINERvA Collaboration], Phys. Rev. Lett. 111, no. 2, 022501 (2013).

[29] U. Mosel, O. Lalakulich and K. Gallmeister, Phys. Rev. D 89, no. 9, 093003 (2014).

[30] A. A. Aguilar-Arevalo et al. [MiniBooNE Collaboration], Phys. Rev. D 81, 013005 (2010).

[31] A. A. Aguilar-Arevalo et al. [MiniBooNE Collaboration], Phys. Rev. D 83, 052007 (2011).

[32] B. Eberly et al. [MINERvA Collaboration], arXiv:1406.6415 [hep-ex].

[33] O. Lalakulich, U. Mosel and K. Gallmeister, Phys. Rev. C 86, 054606 (2012). 